\title{
Effects of sex and racial/ethnic group on the pattern of gastric cancer localization
}

\author{
James C. Yao ${ }^{1}$, Isac I. Schnirer ${ }^{1}$, Satish Reddy ${ }^{1}$, Silvia Chiang ${ }^{1}$, Azmeena Najam ${ }^{1}$, Christina Yu ${ }^{1}$, \\ Geoff Giacco $^{2}$, Keneth Hess ${ }^{3}$, Asif Rashid ${ }^{4}$, Keping Xie ${ }^{1}$, Patrick Lynch ${ }^{5}$, and Jaffer A. Ajani ${ }^{1}$ \\ ${ }^{1}$ Department of Gastrointestinal Medical Oncology, Unit 426, The University of Texas M.D. Anderson Cancer Center, \\ 1515 Holcombe Blvd., Houston, TX 77030, USA \\ ${ }^{2}$ Department of Medical Informatics, The University of Texas M.D. Anderson Cancer Center, Houston, TX, USA \\ ${ }^{3}$ Department of Biomathematics, The University of Texas M.D. Anderson Cancer Center, Houston, TX, USA \\ ${ }^{4}$ Department of Pathology, The University of Texas M.D. Anderson Cancer Center, Houston, TX, USA \\ ${ }^{5}$ Department of Gastrointestinal Medicine and Nutrition, The University of Texas M.D. Anderson Cancer Center, Houston, TX, USA
}

\begin{abstract}
Background. In Western countries, the overall incidence of gastric cancer is declining, while the incidence of proximal gastric cancer is rising. We evaluated the effect of sex, racial/ ethnic group, and age on the localization of gastric cancer.

Methods. The records of all gastric cancer patients who registered at The University of Texas M. D. Anderson Cancer Center from January 1, 1985, to May 31, 1998, were reviewed. Tissue diagnosis and established tumor location were required.

Results. The case records of 1242 eligible patients, consisting of 821 non-Hispanic white, 230 Hispanic, 125 African American, 63 Asian, and 3 Native American patients, were analyzed. Among the 459 women, racial/ethnic group did not have a significant impact on gastric cancer localization $(P=0.57)$. However, among the 783 men, proximal cancers were significantly more frequent in the non-Hispanic white patients $(57 \%$ vs $38 \%$ Hispanic, $41 \%$ African American, 17\% Asian; $P<$ 0.0001). Symptoms at diagnosis significantly varied for proximal vs non-proximal cancers.

Conclusion. Proximal gastric cancer localization was more frequent among non-Hispanic white men, while non-Hispanic white women had a localization pattern similar to those of other racial/ethnic groups. Proximal gastric cancer represents a distinct subtype of gastric cancer that may have different biology, risk factors, and clinical behavior.
\end{abstract}

Key words Stomach · Neoplasms · Epidemiology · Prognosis · Survival

\section{Introduction}

In the United States, the overall incidence of gastric cancer has been on the decline since 1930. However, gastric cancer remains one of the top ten causes of

Offprint requests to: J.A. Ajani

Received: March 1, 2002 / Accepted: August 6, 2002 cancer-related death. Also, during the past decade, a trend of increasing incidence of proximal gastric cancer has been reported by a number of investigators [1-4]. A similar change in the pattern of gastric cancer localization has been observed in Europe and Asia [5-7]. The cause of this increase in the incidence of proximal gastric cancer is under active investigation.

Proximal gastric cancer has a different clinical and biologic behavior from that of gastric cancer in other locations. Specifically, several investigators have reported a worse prognosis for patients who have proximal gastric cancer than for those whose cancer is located in the mid or distal portion of the stomach [6-9]. Additionally, the incidence of proximal gastric cancer is higher among non-Hispanic whites [1], and obesity may play a role in its pathogenesis. For example, in two casecontrol studies conducted in the United States and one conducted in Sweden, an association between a high body mass index and adenocarcinoma of the gastric cardia was observed $[4,10,11]$. Tobacco use has also been reported to increase the risk of proximal gastric cancer [7,10,12,13].

Sex may also play a role in the biology of gastric cancer. In a Korean study involving 10783 patients who had undergone gastric resection, women had a slight but statistically significantly superior 5-year survival rate when compared with men $(57.8 \%$ vs $54.6 \% ; P=0.0175)$ [6]. This finding was confirmed in an analysis of the National Cancer Data Base [9]. In this joint report by the American College of Surgeons and American Cancer Society, Hundahl and colleagues found that women had superior 5-year (31\% vs 25\%) and 10-year survival rates $(24 \%$ vs $18 \%)$ when compared with men.

In the present study, we evaluated the effects of the patient's ethnicity and sex on the location of gastric cancer in patients seen at The University of Texas M. D. Anderson Cancer Center. The effects of gastric cancer localization on symptoms at the time of diagnosis were also examined. 


\section{Patients and methods}

\section{Patient population}

The present study included all gastric cancer patients who registered at M. D. Anderson Cancer Center from January 1, 1985, to May 31, 1998. Each patient's medical record was analyzed. The diagnosis of gastric adenocarcinoma was confirmed, and the tumor location was identified.

\section{Classification and data collection}

Cancer location was established using esophagogastroduodenoscopy or an upper gastrointestinal $X$-ray series, or at surgery. Cancers centered at the esophagogastric junction, gastric cardia, or gastric fundus were classified as proximal, while those centered in the rest of the stomach were classified as nonproximal. Diffuse involvement of the stomach was also classified as nonproximal.

Similar to the classification system used by the Surveillance, Epidemiology, and End Results (SEER) program [14], we divided the patients into five racial/ ethnic groups, non-Hispanic white, Hispanic, African American, Asian, and Native American.

The patients' symptoms at the time of diagnosis were obtained from their records. Weight loss was defined as the loss of more than $10 \%$ of body weight or 10 pounds $(4.54 \mathrm{~kg})$; a description of significant weight loss was also accepted if weight measurements were not available. A database was established, and an oncologist verified all data in the database.

\section{Statistical analysis}

The $\chi^{2}$ test was used to test factors that were associated with gastric cancer localization. The univariate odds ratio (OR) was computed for proximal versus nonproximal gastric cancer location. Additionally, multivariate logistic regression analyses were performed to provide multivariate ORs for factors affecting gastric cancer localization and to adjust for the effect of study factors. Finally, two-sided $P$ values were reported.

\section{Results}

\section{Patient characteristics}

The case records of 1339 consecutive patients who had gastric cancer were examined. This analysis included only 1242 patients (93\%). The reasons for ineligibility in the remaining cases included a lack of proximal to distal location information (69 cases), gastric remnant cancer (23 cases; not classifiable in the proximal to distal orien-

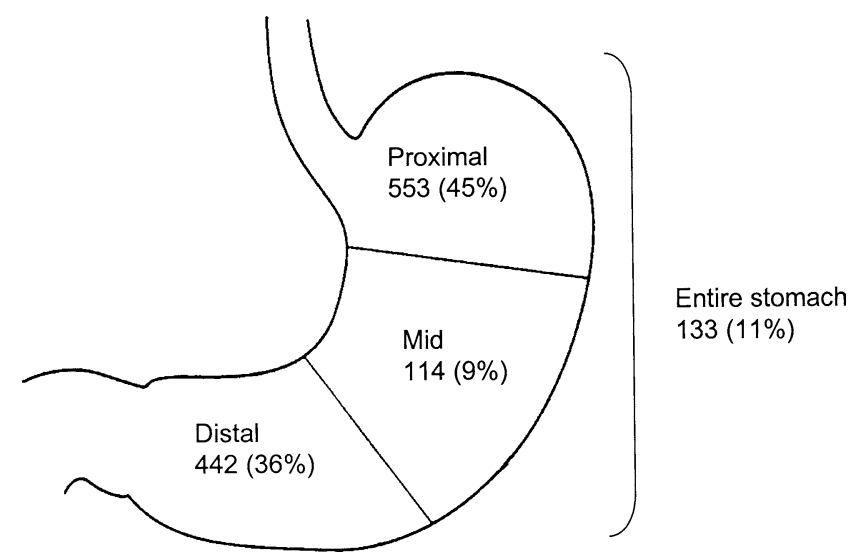

Fig. 1. Distribution of gastric cancer by subsites

Table 1. Patient characteristics $(n=1242)$

\begin{tabular}{|c|c|c|c|c|}
\hline \multirow[b]{2}{*}{ Characteristic } & \multicolumn{2}{|c|}{ Men } & \multicolumn{2}{|c|}{ Women } \\
\hline & $n$ & $(\%)$ & $n$ & $(\%)$ \\
\hline Total no. of patients & 783 & $(63)$ & 459 & $(37)$ \\
\hline \multicolumn{5}{|l|}{ Tumor location } \\
\hline Proximal & 393 & $(50)$ & 160 & $(35)$ \\
\hline Nonproximal & 390 & $(50)$ & 299 & $(65)$ \\
\hline \multicolumn{5}{|l|}{ Ethnicity } \\
\hline White, non-Hispanic & 539 & $(69)$ & 282 & $(61)$ \\
\hline Hispanic & 142 & (18) & 88 & (19) \\
\hline African American & 66 & $(8)$ & 59 & (13) \\
\hline Asian & 35 & (5) & 28 & (6) \\
\hline Native American & 1 & $(0.1)$ & 2 & $(0.4)$ \\
\hline Mean age years $( \pm S D)$ & 58 & $( \pm 14)$ & 56 & $( \pm 15)$ \\
\hline
\end{tabular}

tation), and a lack of racial and ethnicity information (5 cases).

Of the patients included, $783(63 \%)$ were men, and $459(37 \%)$ were women. This is similar to the distribution observed during the same period in the SEER database. The distribution of cancer is illustrated in Fig. 1. A description of the patient characteristics by sex is outlined in Table 1. The median duration from the date of tissue diagnosis to first visit at M. D. Anderson Cancer Center was 18 days.

\section{Symptoms present at time of gastric cancer diagnosis}

Abdominal pain and weight loss were the two most common symptoms present at the time of diagnosis. These occurred in $58 \%$ and $40 \%$ of the patients, respectively. When the symptoms were analyzed according to the location of the cancer within the stomach, the types experienced by the patients were related to the location (Table 2). Specifically, dysphagia occurred predominantly in patients with proximal gastric cancer, while abdominal pain, nausea/vomiting, and early sati- 
Table 2. Symptoms at time of diagnosis by cancer location

\begin{tabular}{lcccc}
\hline Symptoms & $\begin{array}{c}\text { Proximal } \\
(553)\end{array}$ & $\begin{array}{c}\text { Nonproximal } \\
(689)\end{array}$ & $\begin{array}{c}\text { OR } \\
(95 \% \mathrm{CI})^{\mathrm{a}}\end{array}$ & $P$ value \\
\hline Abdominal pain & $276(50 \%)$ & $447(65 \%)$ & $0.5(0.4-0.7)$ & $<0.001$ \\
Weight loss & $222(40 \%)$ & $277(40 \%)$ & $1.0(0.8-1.3)$ & $\mathrm{NS}$ \\
Dysphagia & $210(38 \%)$ & $83(12 \%)$ & $4.5(3.4-6.0)$ & $<0.001$ \\
Nausea/vomiting & $88(16 \%)$ & $182(26 \%)$ & $0.5(0.4-0.7)$ & $<0.001$ \\
Early satiety & $73(13 \%)$ & $132(19 \%)$ & $0.6(0.5-0.9)$ & 0.005 \\
Bleeding & $89(16 \%)$ & $116(17 \%)$ & $0.9(0.7-1.3)$ & $\mathrm{NS}$ \\
\hline
\end{tabular}

OR, Odds ratio; CI, confidence interval; NS, not significant

${ }^{a} \mathrm{OR}$ of proximal gastric cancer patients having symptoms; nonproximal $(\mathrm{OR}=1)$ is referent

Table 3. Proximal gastric cancer localization by sex and ethnicity

\begin{tabular}{lrrr}
\hline Patient groups & Proximal & Nonproximal & $P$ value \\
\hline Men & & & $<0.0001$ \\
White, non-Hispanic & $306(57 \%)$ & $233(43 \%)$ & \\
Hispanic & $54(38 \%)$ & $88(62 \%)$ & \\
African American & $27(41 \%)$ & $39(59 \%)$ & 0.567 \\
Asian & $6(17 \%)$ & $29(83 \%)$ & \\
Women & $105(37 \%)$ & $177(63 \%)$ & \\
White, non-Hispanic & $27(31 \%)$ & $61(69 \%)$ & \\
Hispanic & $18(31 \%)$ & $41(70 \%)$ & \\
African American & $10(36 \%)$ & $18(64 \%)$ & \\
Asian & & & \\
\hline
\end{tabular}

ety were associated with nonproximal gastric cancer. Weight loss and bleeding occurred with similar frequency among proximal and nonproximal gastric cancer patients.

\section{Gastric cancer location}

As there were only three Native Americans present, they were excluded from comparative analyses. Univariate analyses showed that proximal cancer localization was more common among non-Hispanic whites and men. Proximal gastric cancer localization was observed in $50 \%$ of non-Hispanic white patients compared with $25 \%, 36 \%$, and $35 \%$ of Asian, African American, and Hispanic patients $(P<0.001)$.

Proximal gastric cancer localization was observed in $50 \%$ of male patients compared with $35 \%$ of female patients $(P<0.001)$. Age at time of diagnosis was similar between patients with proximal and nonproximal gastric cancer (mean ages of 58 years and 57 years, respectively). When analyzed as a continuous variable by logistic regression, age at time of cancer diagnosis did not affect cancer localization $(P=0.21)$.

Next, we examined the effect of racial/ethnic group on gastric cancer localization among men and women as separate groups. Among the 459 women, racial/ethnic group did not have a significant impact on the frequency of proximal gastric cancer localization. Among the
783 men, proximal cancer localization was significantly more frequent in the non-Hispanic white group $(P<$ 0.0001 ). The effects of racial/ethnic group on gastric cancer localization were thus dependent on patient sex (Table 3).

We also performed logistic regression analyses to adjust for the effects of study factors and to formally analyze the interaction between sex and racial/ethnic group (Table 4). In multivariate analyses, sex and racial/ethnic group independently affected gastric cancer localization. Men were more likely to have proximal gastric cancer than women $(\mathrm{P}<0.001)$. Non-Hispanic white patients were more likely to have proximal gastric cancer localization than Hispanic, African American, and Asian patients $(\mathrm{P}<0.001)$.

When the interaction of racial/ethnic group by sex was added to the model, the effect of racial/ethnic group was dependent on sex $(P=0.021)$. Proximal gastric cancer localization was more frequent among nonHispanic white men. Non-Hispanic white women had a pattern of gastric cancer localization similar to those of the other racial/ethnic groups.

\section{Discussion}

The rising incidence of proximal gastric cancer has wide-reaching clinical implications. Proximal tumors 
Table 4. Multivariate analyses of gastric cancer localization

\begin{tabular}{|c|c|c|c|c|c|}
\hline Patient groups & Proximal & $\begin{array}{c}\text { Univariate } \\
\text { OR }(95 \% \mathrm{CI})\end{array}$ & $P$ value & $\begin{array}{l}\text { Multivariate } \\
\text { OR }(95 \% \text { CI })\end{array}$ & $P$ value \\
\hline \multicolumn{6}{|l|}{ Sex } \\
\hline Women & $35 \%$ & 1.0 & & 1.0 & \\
\hline Men & $50 \%$ & $1.9(1.5-2.4)$ & $<0.001$ & $1.8(1.4-2.3)$ & $<0.001$ \\
\hline \multicolumn{6}{|l|}{ Racial/ethnic group } \\
\hline White, non-Hispanic & $50 \%$ & 1.0 & & 1.0 & \\
\hline Hispanic & $35 \%$ & $0.5(0.4-0.7)$ & $<0.001$ & $0.5(0.4-0.7)$ & $<0.001$ \\
\hline African American & $36 \%$ & $0.6(0.4-0.8)$ & 0.004 & $0.6(0.4-0.9)$ & 0.011 \\
\hline Asian & $25 \%$ & $0.3(0.2-0.6)$ & $<0.001$ & $0.4(0.2-0.6)$ & 0.001 \\
\hline Age (continuous variable) & NA & $1.0(0.997-1.013)$ & 0.21 & $1.0(0.992-1.008)$ & 0.98 \\
\hline
\end{tabular}

OR, Odds ratio; CI, confidence interval; NS, not significant; NA, not applicable

may have different risk factors and potentially different clinical outcomes. Additionally, ethnicity and sex have been reported to affect the biology of gastric cancer $[1,6,9]$. In our study, proximal localization was strikingly more frequent in non-Hispanic white men, while nonHispanic white women had a pattern of gastric cancer localization similar to those of other ethnic-racial groups. Given the poorer prognosis of proximal gastric cancer, the higher proportion of proximal tumors observed in non-Hispanic white men in our study may contribute to the small but consistent survival advantage in women reported by Kim et al. [6] and Hundahl et al. [9].

Our study was conducted at the M. D. Anderson Cancer Center, a major cancer referral center that is thus potentially subject to referral bias. However, several facts make this bias unlikely in the present study. First, the proportion of male to female patients having gastric cancer was similar to the national average [15]. Second, if proximal gastric cancer is defined as cancer of the esophagogastric junction, gastric cardia, or gastric fundus, then the proportion of patients having proximal gastric cancer in the present study is similar to that in other large population-based studies. In the recent report of the National Cancer Data Base by Hundahl et al. [9], 31\% of the patients had cancer of the gastric cardia or fundus, and $38 \%$ of patients did not have their cancers classified in the proximal to distal orientation. Thus, of the patients having gastric cancer classified in this orientation, approximately $50 \%$ had localization in the gastric cardia or fundus. This percentage is similar to the $45 \%$ observed in our study.

Our present study is unique in an important aspect. Previous reports of the clinical behavior of proximal gastric cancer were limited to patients undergoing surgery. However, differing rates of advanced unresectable disease among patients with proximal and nonproximal gastric cancer may bias such studies. In the present study, we included all patients regardless of resectability.
The type of surgical resection used is an area of controversy in the treatment of proximal gastric cancer. Some investigators have advocated the use of total gastrectomy with extensive (D2 or higher) lymphadenectomy for proximal gastric cancer. However, two prospective randomized trials comparing D1 versus D2 lymphadenectomy for gastric cancer, conducted in the Netherlands [16] and England [17], reported much higher rates of operative complications in the D2 group. Further, no improvements in the 5-year survival rates were observed for the D2 group. In the United States, D2 or higher lymphadenectomy is rarely performed [18]. Also, in a recent analysis of 110 cases of proximal gastric cancer, Shimada et al. [19] found that Japanese patients undergoing D2 dissection with total gastrectomy had higher morbidity and mortality rates when compared with patients undergoing D1 dissection with proximal gastrectomy. The investigators recommended D1 dissection with proximal gastrectomy for patients who have a superficial tumor less than $4 \mathrm{~cm}$ in size.

In addition, quality of life after gastrectomy differs depending on the type of gastrectomy performed. In a study of Japanese patients who underwent gastric resection with D2 lymphadenectomy, Korenaga et al. [20] showed that patients had inferior food tolerance, weight, and performance status compared with those who underwent partial gastrectomy. Also, in a British study, Anderson and MacIntyre [21] found that new symptoms (those not present prior to surgical resection) were more frequent after total gastrectomy than after partial gastrectomy or esophagectomy with proximal partial gastrectomy.

The findings described above suggest that proximal gastrectomy may be adequate for $\mathrm{T} 1$ or $\mathrm{T} 2$ proximal gastric carcinoma, resulting in fewer postoperative complications. Newer treatment strategies, such as preoperative chemotherapy or postoperative chemoradiotherapy, as carried out in the Intergroup 0116 trial, may improve outcomes in patients having proximal gastric cancer [22]. 
In conclusion, proximal gastric cancer has different symptoms and types of clinical behavior and may have different risk factors from nonproximal gastric cancer. Current staging systems and treatment strategies do not fully exploit these differences. Further investigations are needed to delineate the clinical and molecular differences between patients with proximal and nonproximal gastric cancer.

Acknowledgment Supported in part by a grant from the Cantu, Caporella, and Smith Families, and River Creek Foundation.

\section{References}

1. Blot WJ, Devesa SS, Kneller RW, Fraumeni JFJ. Rising incidence of adenocarcinoma of the esophagus and gastric cardia. JAMA 1991;265:1287-9.

2. Pera M, Cameron AJ, Trastek VF, Carpenter HA, Zinsmeister AR. Increasing incidence of adenocarcinoma of the esophagus and esophagogastric junction. Gastroenterology 1993;104:510-3.

3. Zheng T, Mayne ST, Holford TR, Boyle P, Liu W, Chen Y, et al. The time trend and age-period-cohort effects on incidence of adenocarcinoma of the stomach in Connecticut from 1955-1989. Cancer 1993;72:330-40.

4. Chow WH, Blot WJ, Vaughan TL, Risch HA, Gammon MD, Stanford JL, et al. Body mass index and risk of adenocarcinomas of the esophagus and gastric cardia. J Natl Cancer Inst 1998;90: 150-5.

5. Powell J, McConkey CC. The rising trend in oesophageal adenocarcinoma and gastric cardia. Eur J Cancer Prev 1992;1:265-9.

6. Kim JP, Lee JH, Kim SJ, Yu HJ, Yang HK. Clinicopathologic characteristics and prognostic factors in 10783 patients with gastric cancer. Gastric Cancer 1998;1:125-33.

7. Inoue M, Tajima K, Hirose K, Kuroishi T, Gao CM, Kitoh T. Lifestyle and subsite of gastric cancer - joint effect of smoking and drinking habits. Int J Cancer 1994;56:494-9.

8. Wanebo HJ, Kennedy BJ, Chmiel J, Steele G, Jr., Winchester D, Osteen R. Cancer of the stomach. A patient care study by the American College of Surgeons. Ann Surg 1993;218:583-92.

9. Hundahl SA, Phillips JL, Menck HR. The National Cancer Data Base Report on poor survival of U.S. gastric carcinoma patients treated with gastrectomy: fifth edition American Joint Committee on Cancer staging, proximal disease, and the "different disease" hypothesis. Cancer 2000;88:921-32.

10. Vaughan TL, Davis S, Kristal A, Thomas DB. Obesity, alcohol, and tobacco as risk factors for cancers of the esophagus and gastric cardia: adenocarcinoma versus squamous cell carcinoma. Cancer Epidemiol Biomarkers Prev 1995;4:85-92.

11. Lagergren J, Bergstrom R, Nyren O. Association between body mass and adenocarcinoma of the esophagus and gastric cardia. Ann Intern Med 1999;130:883-90.

12. Lagergren J, Bergstrom R, Lindgren A, Nyren O. The role of tobacco, snuff and alcohol use in the aetiology of cancer of the oesophagus and gastric cardia. Int J Cancer 2000;85:340-6.

13. Gammon MD, Schoenberg JB, Ahsan H, Risch HA, Vaughan TL, Chow WH, et al. Tobacco, alcohol, and socioeconomic status and adenocarcinomas of the esophagus and gastric cardia. J Natl Cancer Inst 1997;89:1277-84.

14. Miller BA, Kolonel LN, National Cancer Institute (US). Cancer Control Research Program. Racial/ethnic patterns of cancer in the United States, 1988-1992. Washington, DC: US Department of Health and Human Services National Institute of Health; 1996.

15. Greenlee RT, Murray T, Bolden S, Wingo PA. Cancer statistics, 2000. CA Cancer J Clin 2000;50:7-33.

16. Bonenkamp JJ, Hermans J, Sasako M, van de Velde CJ. Extended lymph-node dissection for gastric cancer. Dutch Gastric Cancer Group. N Engl J Med 1999;340:908-14.

17. Cuschieri A, Weeden S, Fielding J, Bancewicz J, Craven J, Joypaul V, et al. Patient survival after D1 and D2 resections for gastric cancer: long-term results of the MRC randomized surgical trial. Surgical Co-operative Group. Br J Cancer 1999;79:152230.

18. Estes NC, MacDonald JS, Touijer K, Benedetti J, Jacobson J. Inadequate documentation and resection for gastric cancer in the United States: a preliminary report. Am Surg 1998;64:680-5.

19. Shimada H, Suzuki T, Nakajima K, Hori S, Hayashi H, Takeda A, et al. Lymph node metastasis with adenocarcinoma of the gastric cardia: clinicopathological analysis and indication for D1 dissection. Int Surg 1999;84:13-7.

20. Korenaga D, Orita H, Okuyama T, Moriguchi S, Maehara Y, Sugimachi K. Quality of life after gastrectomy in patients with carcinoma of the stomach. Br J Surg 1992;79:248-50.

21. Anderson ID, MacIntyre IM. Symptomatic outcome following resection of gastric cancer. Surg Oncol 1995;4:35-40.

22. Macdonald JS, Smalley S, Benedetti J, Estes N, Haller DG, Ajani JA, et al. Postoperative combined radiation and chemotherapy improves disease-free survival (DFS) and overall survival (OS) in resected adenocarcinoma of the stomach and G.E. junction. Results of Intergroup study INT-0116 (SWOG 9008). Proc Am Soc Clin Oncol 2000;19:1a. 